University of Nebraska - Lincoln

DigitalCommons@University of Nebraska - Lincoln

Faculty Publications from the Department of Electrical \& Computer Engineering, Department Electrical and Computer Engineering

2011

\title{
Non-Binary Joint Network-Channel Decoding of Correlated Sensor Data in Wireless Sensor Networks
}

\author{
Arindra Guha \\ University of Nebraska-Lincoln, arindra.guha@huskers.unl.edu \\ Eric T. Psota \\ University of Nebraska-Lincoln, epsota@unl.edu \\ Lance C. Pérez \\ University of Nebraska-Lincoln, Iperez@unl.edu
}

Follow this and additional works at: https://digitalcommons.unl.edu/electricalengineeringfacpub

Part of the Electrical and Computer Engineering Commons

\begin{abstract}
Guha, Arindra; Psota, Eric T.; and Pérez, Lance C., "Non-Binary Joint Network-Channel Decoding of Correlated Sensor Data in Wireless Sensor Networks" (2011). Faculty Publications from the Department of Electrical and Computer Engineering. 215.

https://digitalcommons.unl.edu/electricalengineeringfacpub/215
\end{abstract}

This Article is brought to you for free and open access by the Electrical \& Computer Engineering, Department of at DigitalCommons@University of Nebraska - Lincoln. It has been accepted for inclusion in Faculty Publications from the Department of Electrical and Computer Engineering by an authorized administrator of DigitalCommons@University of Nebraska - Lincoln. 


\title{
Non-Binary Joint Network-Channel Decoding of Correlated Sensor Data in Wireless Sensor Networks
}

\author{
Arindra Guha, Eric T. Psota, and Lance C. Pérez \\ Department of Electrical Engineering \\ University of Nebraska, Lincoln, NE 68588 \\ Email: arindra.guha@huskers.unl.edu, epsota24@huskers.unl.edu,lperez@unl.edu
}

\begin{abstract}
The performance of non-binary joint networkchannel decoding (NB-JNCD) is examined in wireless sensor networks with correlated sensors. The operating assumption is that systems containing multiple sensors in close proximity obtain correlated measurements. It is shown that, when the correlation model between sensors is known at the sink, the addition of a correlationbased decoder within the decoding framework greatly improves the error rate performance. Simulation results also indicate that the proposed correlation-based decoder is comparable to source coding across the correlated sensors in terms of error rate, while eliminating the need for costly sensor-to-sensor communication.
\end{abstract}

\section{INTRODUCTION}

Network coding (NC) was first proposed by Ahlswede et al. in 2000 [1]. Network coding improves data rates, when compared to traditional routing methods, by intelligently combining data in order to achieve the information transmission capacity proposed by the max-flow min-cut theorem. Due to its capacityachieving performance in multicast wireline networks, network coding has attracted much attention in the research community.

The utility of network coding reaches beyond wireline communications, and can be applied to a variety of wireless applications. Much of the network coding research in wireless networks assumes lossless channels [2], [3], [4], however, research has also demonstrated the utility of network coding on lossy network applications [5], [6], [7]. One such application is wireless sensor networks (WSN), which can be used to collect data in a variety of industrial and civilian applications, including industrial process monitoring [8], and health monitoring [9].

Channel coding is commonly used to improve reliability over point-to-point lossy communications links, so it is natural to consider the application of channel coding with network coding over lossy networks to improve link reliability and increase the transmission rate. Effros et al. [10] conjectured that channel and network coding do not separate in general for wireless networks and attributes this to the failure of separation in canonical systems. They go on to demonstrate improved performance using a unified approach, which was verified by [11].

Hausl et al. first proposed a joint network-channel decoding technique for multi-access and two-way relay channels, and presented an iterative network and channel decoding framework that operates on the Tanner graph of the LDPC code [12], [13]. Bao and Li proposed adaptive network coded cooperation using multiple transmitters to send data to a common receiver through an inherently unreliable and constantly changing channel [14]. They also presented a general framework that unifies channel coding and network coding [15]. The above schemes are based on networks with specific topologies using binary symbols, and cannot be directly applied to general wireless network topologies with non-binary coding. More recently, non-binary joint network-channel decoding (NB-JNCD) [16], [17] has been introduced as a practical scheme that can be applied to general network topologies (e.g., wireless sensor networks) by combining non-binary channel coding and random linear network coding using high-order modulation schemes for improved bandwidth efficiency. An iterative two-tier decoding scheme is used by NB-JNCD to jointly exploit redundancy inside packets (channel coding) and across packets (network coding) for error recovery. Simulation results show significant error-rate improvements when comparing NB-JNCD to similar decoding methods.

This paper focuses on the application of NB-JNCD to networks with correlated sensors, e.g., wireless body area networks (WBAN). WBANs consists of sensors that are connected either inside or outside of the human body, and can be used to monitor vital body parameters [9], [18]. The WBAN measurements obtained from the same subject, such as blood pressure, heart beat, and pulse rate, are likely to be correlated. While source coding can reduce the necessary data rate of individual sensors by exploiting temporal correlation, sensorto-sensor communication is necessary to apply source coding across sensors. To eliminate the need for increased power and complexity at the sensor nodes, a new non-binary joint channel-correlation-network decoder (NB-JCCND) is presented. By assuming known correlation across sensors, the NB-JCCND incorporates a new correlation-based decoding component to reduce the error rate as the correlation between sensors increases. In addition NB-JCCND is also compared to a system which performs source coding across sensors and uses NB-JNCD. It is shown that NB-JCCND reduces the complexity at the sensors, otherwise incurred by sensor-to-sensor source coding, without resulting in significant error rate increases.

\section{BACKGROUND}

The non-binary joint network-channel-correlation decoder uses a combination of LDPC decoding, linear network coding, and a correlation-based message-passing algorithm. The following review of LDPC codes and random linear network coding is given before reviewing the NB-JNCD scheme of [17].

\section{A. LDPC Codes}

Low-density parity-check codes were first introduced by Gallager in 1960 [19], and rediscovered by MacKay in 1997 [20]. This rediscovery was brought about due to powerful computational resources, and LDPC codes were shown to achieve 
near-Shannon limit performance when decoded using iterative, probabilistic decoding algorithms.

This paper considers the following network communication model, using non-binary LDPC codes [21] to improve the reliability of data transmission in wireless sensor networks. For a single data packet transmission, each sensor generates a vector $\mathbf{u} \in \mathbb{F}_{q}^{K}$ of $K$ independent information symbols, where the symbols are elements of the Galois field of size $q=2^{m}$. A channel encoder uses a generator matrix $\mathbf{G}$ to map $\mathbf{u}$ to a unique length $N$ codeword $\mathbf{c} \in \mathbb{F}_{q}^{N}$ via $\mathbf{c}=\mathbf{u G}$. The rate of the resulting LDPC code is $R=\frac{K}{N}$.

Before a codeword $\mathbf{c} \in C$ is transmitted over a noisy channel, each symbol is mapped to a point using either binary phase-shift keying (BPSK) or quadrature amplitude modulation (QAM). The received signal vector $\mathbf{y} \in \mathbb{R}^{N}$ is given by $\mathbf{y}=\mathbf{x}+\mathbf{n}$, where $\mathbf{n} \in \mathbb{R}^{N}$ is a vector of additive white Gaussian noise (AWGN) with variance $\sigma^{2}$. The channel likelihood vector, often used for soft-decision decoding is given by

$$
\lambda_{i, j}=\frac{e^{\frac{-\left\|y_{i}-x_{j}\right\|^{2}}{2 \sigma^{2}}}}{\sum_{k=0}^{q-1} e^{\frac{-\left\|y_{i}-x_{k}\right\|^{2}}{2 \sigma^{2}}}}
$$

for all $i=1, \ldots, N$, and $j=0, \ldots, q-1$. Note that $\left\|y_{i}-x_{j}\right\|$ is the Euclidean distance between the noisy modulated symbol $y_{i}$ and the modulated information symbol $x_{j}$.

For any given generator matrix $\mathbf{G}$, there exists an $M \times N$ parity-check matrix $\mathbf{H}$, where $M \geq(N-K)$. A parity-check matrix of an LDPC code $C$ is a sparse matrix $\mathbf{H}$, such that $\mathbf{H c}^{T}=\mathbf{0}$ for all $\mathbf{c} \in C$. LDPC codes are often defined by $\mathbf{H}$, and the Tanner graph is a common bipartite graphical representation of $\mathbf{H}$, used to depict the operations of messagepassing decoders. To construct the Tanner graph, each column $i$ in $\mathbf{H}$ is assigned to a corresponding variable node $v_{i} \in V$, and each row $j$ is assigned to a corresponding check node $f_{j} \in F$. An edge $e_{i, j} \in E$ exists between $v_{i}$ and $f_{j}$ if and only if the entry in the $j^{\text {th }}$ row and $i^{\text {th }}$ column of $\mathbf{H}$ is non-zero.

Belief propagation decoding is used in this paper for iterative decoding of LDPC codes. After computing the initial likelihood of each symbol using Equation (1), messages are passed from variable nodes to check nodes, and check nodes to variable nodes for a pre-determined number of iterations. Further details regarding the decoding algorithm for non-binary LDPC codes used in this paper can be found in [21].

\section{B. Network Coding}

Network coding operates by intelligently combining data at intermediate nodes [1]. This is in contrast to traditional data routing that tries to avoid collisions of data streams by simply replicating data and forward it. The principles of network coding are examined using the network given in Figure 1. Two bits are being transmitted from the sensor $S$ to the receivers $T_{1}$ and $T_{2}$ in a single unit of time. With a simple encoding function at node $R_{3}$, both nodes $T_{1}$ and $T_{2}$ are able to decode bits $b_{1}$ and $b_{2}$ in a single transmission. Generalized, random, linear network coding [22] is examined in the following.

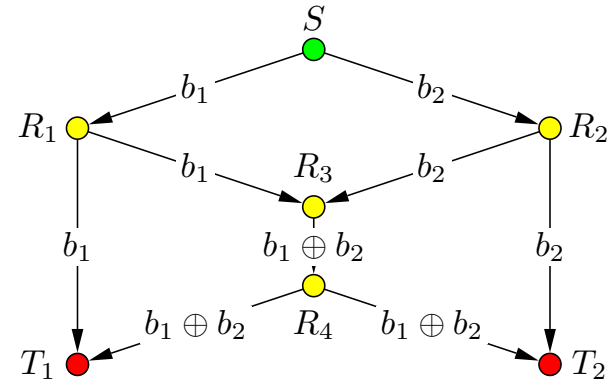

Fig. 1. Network configuration with a simple network coding scheme used to achieve the capacity of two bits per channel transmission to both sinks.

1) Encoding Network Codes: Assume there are $\mathcal{S}$ sensors in the network, and each sensor wishes to transmit a packet $D$ of information symbols. The entire set of packets being transmitted over the network is given by $\left\{D^{1}, \ldots, D^{\mathcal{S}}\right\}$. Intermediate nodes in the network receive a set of $k$ encoded packets, each given by $E_{j}=\sum_{i=1}^{\mathcal{S}} g_{j}^{i} D^{i}$. The intermediate nodes then re-encode the messages using

$$
E_{\text {out }}=\sum_{i=1}^{k} h_{i} E_{i}=\sum_{i=1}^{k}\left(\sum_{j=1}^{\mathcal{S}} h_{i} g_{i}^{j} D^{j}\right)
$$

In order to make network coding possible, the vector of encoding coefficients $\left\{g_{\text {out }}^{1}, \ldots, g_{\text {out }}^{\mathcal{S}}\right\}$, where $g_{\text {out }}^{j}=\sum_{i=1}^{k} h_{i} g_{i}^{j}$ for all $j=1, \ldots, \mathcal{S}$, is assumed to be known by all downstream nodes in the network. In practice, this is done by adding a header to the encoded information vector $E_{\text {out }}$. This operation is performed at all intermediate nodes in the network.

2) Decoding Network Codes: Much like the intermediate nodes, the sink receives a set of encoded packets $\left\{E_{j}=\right.$ $\left.\sum_{i=1}^{\mathcal{S}} g_{j}^{i} D^{i} \mid j=1, \ldots, k\right\}$. In order for the sink to decode the information packets $\left\{D^{1}, \ldots, D^{\mathcal{S}}\right\}$, the number of received packets must satisfy the condition $k \geq \mathcal{S}$. Decoding involves forming a matrix of equations $E_{j}=\sum_{i=1}^{\mathcal{S}} g_{j}^{i} D^{i}$ for all $j=$ $1, \ldots, k$ using knowledge of $\left\{E_{1}, \ldots, E_{k}\right\}$ and the coefficients, and then solving for the information packets $\left\{D^{1}, \ldots, D^{\mathcal{S}}\right\}$ using Gaussian elimination.

\section{Non-Binary Joint Network-Channel Coding}

Non-binary joint network-channel decoding (NB-JNCD) combines channel coding and network coding by introducing an iterative decoder that passes messages between a non-binary LDPC decoder and a random linear network decoder [16], [17]. Both LDPC coding and network coding operate over the same Galois field $G F(q)$.

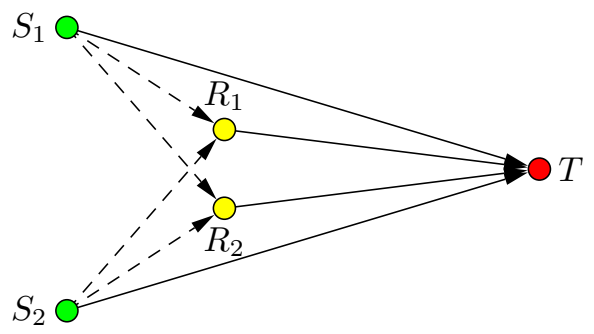

Fig. 2. Two-sensor, one-sink network with two intermediate nodes. 
As an example of NBJNCC, we consider a simple topology of Figure 2, using two sensors $S_{1}$ and $S_{2}$, two intermediate nodes $R_{1}$ and $R_{2}$, and one sink $T$. We use a non-binary LDPC code specified by the parity-check matrix $\mathbf{H}$ and generator matrix G. Sensor $S_{1}$ generates a packet $\mathbf{u}_{1} \in \mathbb{F}_{q}^{K}$ of $K$ information symbols, then encodes it into the packet $D_{1}=\mathbf{u}_{1} \mathbf{G} \in \mathbb{F}_{q}^{N}$. Thus, the channel coding rate is $R_{c}=\frac{K}{N}$. Correspondingly, the packet from sensor $S_{2}$ is $D_{2}=\mathbf{u}_{2} \mathbf{G} \in \mathbb{F}_{q}^{N}$. Packets $D_{1}$ and $D_{2}$ are modulated and sent to both the intermediate nodes and the sink. We assume the channels between the sensors and intermediate nodes are lossless, and all channels connecting to the sink are lossy with slow Rayleigh fading. It is assumed that the fading remains constant across each packet, and varies from packet to packet independently. The Rayleigh block-fading channel is modeled as $\mathbf{y}=\alpha \mathbf{x}+\mathbf{n}$ where $\alpha$ follows a standard Rayleigh distribution with $E\left(|\alpha|^{2}\right)=1$ and $\mathbf{n}$ is AWGN.

After receiving packets from the sensors, the intermediate nodes encode the packets using random linear network coding. Thus, the two network codes transmitted from the intermediate nodes $R_{1}$ and $R_{2}$ to the sink $T$ are

$$
E_{1}=g_{1}^{1} \mathbf{u}_{1} \mathbf{G}+g_{1}^{2} \mathbf{u}_{2} \mathbf{G}
$$

and

$$
E_{2}=g_{2}^{1} \mathbf{u}_{1} \mathbf{G}+g_{2}^{2} \mathbf{u}_{2} \mathbf{G} .
$$

where the set of network coding coefficients $\left\{g_{1}^{1}, g_{1}^{2}, g_{2}^{1}, g_{2}^{2}\right\}$ are chosen randomly from $G F(q)$. The sink receives $D_{1}$ and $D_{2}$ directly from the sensors and network codes $E_{1}$ and $E_{2}$ from the intermediate nodes. Altogether, the information received at the sink node is given by

$$
\left(\begin{array}{c}
D_{1} \\
D_{2} \\
E_{1} \\
E_{2}
\end{array}\right)=\left(\begin{array}{cc}
1 & 0 \\
0 & 1 \\
g_{1}^{1} & g_{1}^{2} \\
g_{2}^{1} & g_{2}^{2}
\end{array}\right)\left(\begin{array}{c}
D_{1} \\
D_{2}
\end{array}\right)=\left(\begin{array}{cc}
1 & 0 \\
0 & 1 \\
g_{1}^{1} & g_{1}^{2} \\
g_{2}^{1} & g_{2}^{2}
\end{array}\right)\left(\begin{array}{c}
\mathbf{u}_{1} \\
\mathbf{u}_{2}
\end{array}\right) \mathbf{G} .
$$

The likelihood vectors for the packets received at the sink can be computed using Equation (1), and are given by $\lambda_{D_{1}}$, $\boldsymbol{\lambda}_{D_{2}}, \boldsymbol{\lambda}_{E_{1}}$, and $\boldsymbol{\lambda}_{E_{2}}$. In the NB-JNCC decoder, the channel codes are first decoded using belief propagation decoding for a fixed number of iterations. Then, the new likelihoods $\lambda_{D_{1}}^{\mathrm{BP}}, \boldsymbol{\lambda}_{D_{2}}^{\mathrm{BP}}$, $\lambda_{E_{1}}^{\mathrm{BP}}$, and $\boldsymbol{\lambda}_{E_{2}}^{\mathrm{BP}}$ are taken from the belief propagation decoder, and used by the network decoder.

Message-passing within the network decoder operates by exchanging information between decoded packets. In this example, each packet in the set $\left\{D_{1}, D_{2}, E_{1}, E_{2}\right\}$ can be formed from a linear combination of any other two, forming the basis for the NB-JNCD scheme. For example, in the simplest case packet $E_{1}$ can be formed using $E_{1}=g_{1}^{1} D_{1}+g_{1}^{2} D_{2}$. The same linear operations can be applied to the likelihoods $\lambda_{D_{1}}^{\mathrm{BP}}$ and $\boldsymbol{\lambda}_{D_{2}}^{\mathrm{BP}}$ to form $\boldsymbol{\lambda}_{E_{1}}^{\mathrm{ND}}$, which is the likelihood produced by network decoding. When updating the likelihood of any packet, the two other packets are chosen that have the smallest number of non-zero elements in their syndrome after BP decoding. Packets that result in a small number of non-zero elements in their syndrome are considered the most reliable. For example, consider a situation where the number of nonzero elements in the syndromes of packets $\left\{D_{1}, D_{2}, E_{1}, E_{2}\right\}$ is $\{23,13,5,28\}$. Under this scenario, when updating the likelihood of $D_{2}$, the likelihoods of $D_{1}$ and $E_{1}$ would be used because, excluding
$D_{2}$, they have the smallest number on non-zero elements in their syndrome. The final step in NB-JNCD is to multiply the ND likelihoods by the original likelihoods obtained from the channel, and normalize them. The result of this multiplication is then passed back into the BP decoder for further processing until all the packets are decoded with all-zero syndromes or up to a fixed number of iterations.

\section{JOINT DeCOding OF CORRElated SENSOR DATA}

This section introduces a new method for joint decoding at the sink node of a wireless sensor network with correlated binary sensors. Using a known model of correlation, the relationship between the sensors is used to achieve lower error rates when compared to using NB-JNCD.

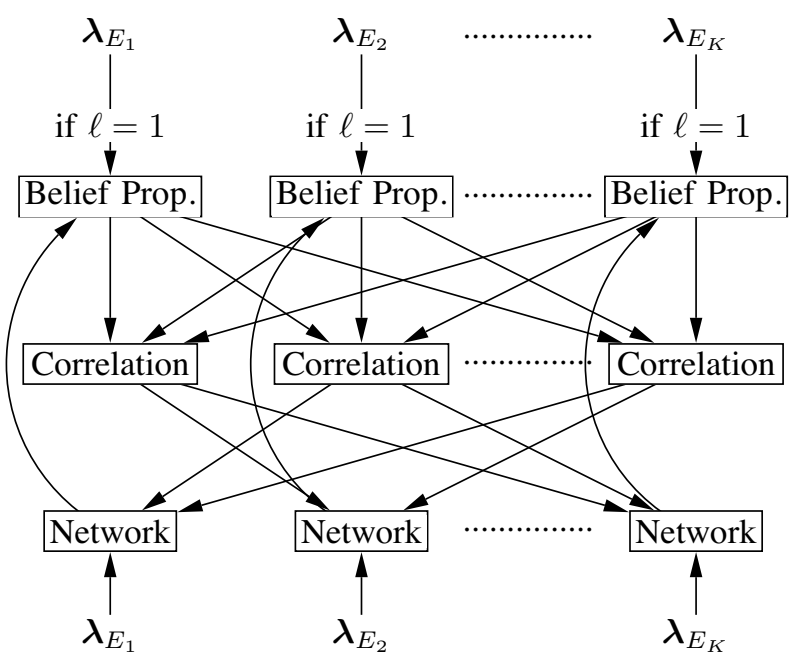

Fig. 3. Non-binary joint channel-correlation-network decoder.

The NB-JCCND, shown in Figure 3, first computes the channel likelihood using Equation (1), and performs belief propagation decoding on each packet separately. The soft output of the belief propagation algorithm is then fed into the correlation decoder, where the known correlation between packets is used to modify the probability of each symbol. These modified probabilities are then passed to the network decoder, which utilizes the redundancy in the network to pass information between packets. The main contribution of this paper is the use of correlation between the sensors in the decoding process. After presenting the correlation model considered in this paper, a detailed description of the NB-JCCND is given.

\section{A. Correlation Model}

The correlation model is given in the following for a twosensor system. Let $\mathbf{u}_{b, 1}=\left[u_{1}^{1}, \ldots, u_{1}^{K m}\right] \in \mathbb{F}_{2}^{K m}$ and $\mathbf{u}_{b, 2}=$ $\left[u_{2}^{1}, \ldots, u_{b, 2}^{K m}\right] \in \mathbb{F}_{2}^{K q}$ be two correlated binary information bit sequences. The correlation model between the two sensors is given by the following [23].

- Generate the symmetric i.i.d. sequence $\mathbf{u}_{b, 1}$, where $P\left(u_{k}^{1}=0\right)=P\left(u_{k}^{1}=1\right)=\frac{1}{2}$ for all $k=1, \ldots, K m$.

- Let e be a binary random vector, such that $P\left(e_{k}=1\right)=$ $1-p_{1,2}$ and $P\left(e_{k}=0\right)=p_{1,2}$ for all $k=1, \ldots, K m$. Define the sequence $\mathbf{u}_{b, 2}$ as $\mathbf{u}_{b, 2}=\mathbf{u}_{b, 1} \oplus \mathbf{e}$, where $\oplus$ indicates modulus 2 addition. 
It is easy to see that, when $p_{1,2}=1.0$, the information bits $\mathbf{u}_{b, 1}=\mathbf{u}_{b, 2}$. This represents two sensors that are completely correlated. The opposite scenario occurs when $p_{1,2}=0.5$, and the two information bits are completely uncorrelated.

Sensor $S_{1}$ transmits $\mathbf{u}_{1}$ of $K$ information symbols using the encoding function $D_{1}=\mathbf{u}_{1} \mathbf{G}$, and similarly sensor $S_{2}$ sends $D_{2}=\mathbf{u}_{2} \mathbf{G}$. The symbols sequences $\mathbf{u}_{1}$ and $\mathbf{u}_{2}$ are obtained by converting sequential groups of $m$ information bits in $\mathbf{u}_{b, 1}$ and $\mathbf{u}_{b, 2}$. It is assumed that $\mathbf{G}$ is systematic, and thus the last $K$ symbols in the codeword are the information symbols. While the information symbols are correlated using the above definition, it is assumed that the coded symbols are uncorrelated. The correlation model for two sensors can easily be extended to any number of sensors, as it is assumed that the correlation probability $p_{i, j}$ is known between any two sensors $S_{i}$ and $S_{j}$.

\section{B. Non-Binary Joint Channel-Correlation-Network Decoder}

1) Channel Decoding: Let there be $k \geq \mathcal{S}$ noisy packets received at the sink, given by $\left\{\mathbf{y}_{E_{1}}, \mathbf{y}_{E_{2}}, \ldots \mathbf{y}_{E_{k}}\right\}$, where each unmodulated, uncorrupted packet $j$ is given by

$$
E_{j}=g_{j}^{1} \mathbf{u}_{1} \mathbf{G}+g_{j}^{2} \mathbf{u}_{2} \mathbf{G}+\ldots+g_{j}^{\mathcal{S}} \mathbf{u}_{\mathcal{S}} \mathbf{G} .
$$

The channel likelihood vector for $\mathbf{y}_{E_{j}}$, given by $\boldsymbol{\lambda}_{E_{j}}$, is computed using equation (1) and passed to the belief propagation decoder, for all $j=1, \ldots, k$. The belief propagation decoder performs a fixed number of iterations, and the soft output of the belief propagation decoder is then passed to the correlation decoder.

2) Correlation Decoding: Correlation decoding exploits the known correlation between sensors by modifying the probability of each symbol after belief propagation decoding. Whereas belief propagation decoder uses relationships between symbols in the same packet to determine the value of each symbol, correlation decoding uses the relationship between symbols across sensors to determine the value of each symbol. The procedure for passing messages between packets using the correlation decoder is given in the following.

\section{- Conditional probability tables}

Before correlation decoding can take place, the conditional probability tables for every pair of packets received at the sink node must be computed. The tables are used to compute the messages passed between packets. Consider two packets $S_{i}$ and $S_{j}$, with correlation probability $p_{i, j}$. The probability that $S_{i}$ transmits a symbol $s_{t} \in\{0, \ldots, q-1\}$ given that sensor $S_{l}$ transmits a symbol $s_{v} \in\{0, \ldots, q-1\}$ is given by

$$
p_{i, j}^{G F}\left(s_{t} \mid s_{v}\right)=\prod_{r=1}^{m} \begin{cases}p_{i, j} & \text { if } b_{t, r}=b_{v, r} \\ 1-p_{i, j} & \text { if } b_{t, r} \neq b_{v, r},\end{cases}
$$

where $b_{t, r}$ and $b_{v, r}$ for $r=1, \ldots, m$ are the bits representing the symbols $s_{t}$ and $s_{v}$, respectively. The conditional probability table between sensor $S_{i}$ and $S_{j}$ contains $p_{i, j}^{G F}\left(s_{t} \mid s_{v}\right)$ for all $s_{t} \in\{0, \ldots, q-1\}$ and $s_{v} \in\{0, \ldots, q-1\}$. A conditional probability table is computed for all $2 \times\left(\begin{array}{c}\mathcal{S} \\ 2\end{array}\right)$ pairs of sensors. We will denote the conditional probability table for every pair of encoded packets $E_{i}$ and $E_{j}$ by $P_{E_{i} \mid E_{j}}$.

\section{- Generate modified likelihoods}

The second step is to modify the soft output of belief propagation using the conditional probability tables. This is done by multiplying the soft output probability vector element-by-element by the conditional probability table. Consider two likelihood vectors passed from the BP decoder for the same symbol, given by $\lambda_{E_{i}}^{\mathrm{BP}}$ and $\lambda_{E_{j}}^{\mathrm{BP}}$. There are two steps to compute the message passed from $\lambda_{E_{i}}^{\mathrm{BP}}$ to $\lambda_{E_{j}}^{\mathrm{BP}}$. The first step is to multiply each element in the probability table by $\lambda_{E_{i}}^{\mathrm{BP}}$ using

$$
p_{i \rightarrow j}^{G F}\left(s_{t}, s_{v}\right)=\lambda_{E_{i}, k}^{\mathrm{BP}} \times p_{i, j}^{G F}\left(s_{t} \mid s_{v}\right)
$$

for all $t=1, \ldots, q$ and $v=1, \ldots, q$. This operation uses the likelihoods obtained from BP decoding to convert the conditional probabilities to joint probabilities. The second step is to sum the elements in the joint probability table to compute the correlation decoder (CD) message, given by

$$
\lambda_{E_{i} \rightarrow E_{j}}^{\mathrm{CD}}=\sum_{v=0}^{q-1} p_{i \rightarrow j}^{G F}\left(s_{t}, s_{v}\right) .
$$

For all correlated packets $E_{i}$ and $E_{j}$, the messages $\lambda_{E_{i} \rightarrow E_{j}}^{\mathrm{CD}}$ and $\lambda_{E_{j} \rightarrow E_{i}}^{\mathrm{CD}}$ are computed. Then, the output of the correlation decoder is found by multiplying each of the extrinsic messages being computed for $E_{i}$. The final output of the correlation decoder is given by

$$
\lambda_{E_{i}}^{\mathrm{CD}}=\prod_{j=1}^{k} \lambda_{E_{j} \rightarrow E_{i}}^{\mathrm{CD}}
$$

where the vectors $\lambda_{E_{j} \rightarrow E_{i}}$ are multiplied element-byelement. In summary, the correlation decoder uses the conditional probability tables to pass information between codewords received at the sink.

3) Network Decoding: The network decoding portion of the NB-JCCND is done using the same approach described in Section II-C, and introduced in [17].

\section{RESUlTS}

The following simulation results demonstrate the performance of the proposed NB-JCCND on the network topology given in Figure 2. Network encoding at the intermediate nodes is performed using the coefficients $g_{1}^{1}=7, g_{1}^{2}=7, g_{2}^{1}=12$, and $g_{2}^{2}=13$. Simulations results are presented using six different sensor correlations $p_{1,2}=0.5,0.6,0.7,0.8,0.9$, and 1.0. An LDPC code with length $N=1000$, dimension $K=800$ symbols over $G F(16)$ is used for all simulations. This is the same LDPC code used in [17], with average variable node degree 2.8. The results are compared to those of NB-JNCD over a range of SNR on the Rayleigh block fading channel. Note that all performance plots take into account the rate of the channel code given by $\frac{K}{N}=0.8$, and the network transmission rate of 0.5 (transmitting 2 symbols per channel use on a network with a capacity of 4), for an overall transmission rate of 0.4 information bits per channel use.

It was determined empirically that the raw likelihoods passed from the correlation decoder to the network decoder were too strong for these simulations, so dampening of the correlation 


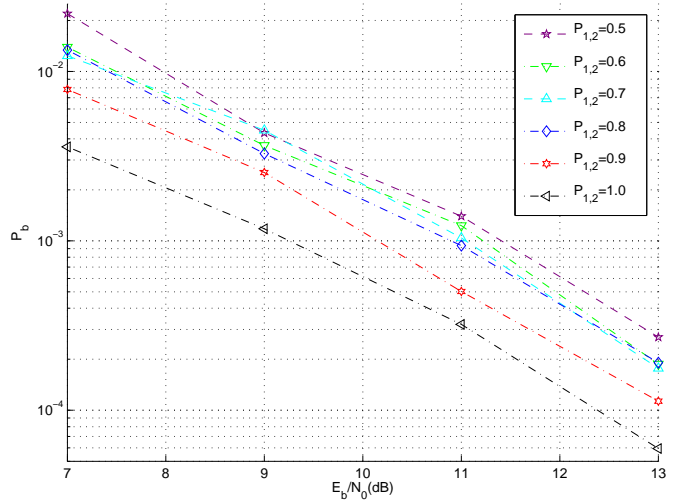

Fig. 4. Probability of bit error for NB-JCCND over the Rayleigh blockfading channel on a two-source, single-sink network under different bit-level correlations.

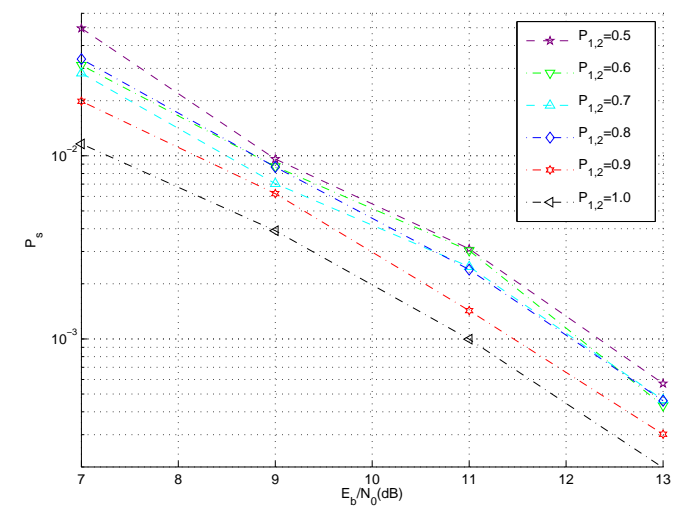

Fig. 5. Probability of symbol error for NB-JCCND over the Rayleigh blockfading channel on a two-source, single-sink network under different bit-level correlations.

decoder output was applied. During simulations over the fading channel, the likelihoods $\boldsymbol{\lambda}_{E_{i}}$ are dampened by passing $\left(2.0 \times \boldsymbol{\lambda}_{E_{1}}^{\mathrm{BP}}+\boldsymbol{\lambda}_{E_{1}}^{\mathrm{CD}}\right) / 3.0$ to the network decoder. Over the AWGN channel, the likelihoods $\boldsymbol{\lambda}_{E_{i}}$ are dampened by passing $\left(4.0 \times \boldsymbol{\lambda}_{E_{1}}^{\mathrm{BP}}+\boldsymbol{\lambda}_{E_{1}}^{\mathrm{CD}}\right) / 5.0$ to the network decoder. The reason for this dampening is as follows. Often, several iterations of NBJCCND are required for successful decoding. By applying raw correlation decoding several times, the correlation across the different sensors is reinforced too often, resulting in an inflated estimate of the correlation that prevents channel decoding from converging. At high SNR, where simulation results are presented for the fading channel, a small number of iterations are typically required, and thus less dampening is required. For AWGN channel simulations at low SNR, more dampening is required due to the increase in the number of iterations.

Both the probability of bit error $\left(P_{b}\right)$ and symbol error $\left(P_{s}\right)$ are shown in Figures 4 and 5. As correlation increases, the error rates of NB-JNCD, with performance equal to NB-JCCND at $p_{1,2}=0.5$, remains unchanged while the error rates of NB-JCCND experience a significant decrease. At $p_{1,2}=1.0$, the NB-JCCND reaches $P_{b}=10^{-3}$ at approximately SNR = 5.2dB, while NB-JNCD does not reach $P_{b}=10^{-3}$ until SNR $=7.4 \mathrm{~dB}$. This corresponds to a $2.2 \mathrm{~dB}$ gain in performance when the sensor data is completely correlated. The gain for $p_{1,2}=0.6,0.7,0.8$, and 0.9 is $0.2 \mathrm{~dB}, 0.4 \mathrm{~dB}, 0.5 \mathrm{~dB}$, and $1.3 \mathrm{~dB}$, respectively.

The symbol error rate gains are slightly less than those achieved with bit error rate. At $P_{s}=3 \times 10^{-3}$, the gain at $p_{1,2}=0.6,0.7,0.8,0.9$, and 1.0 is $0.2 \mathrm{~dB}, 0.3 \mathrm{~dB}, 0.3 \mathrm{~dB}, 0.9 \mathrm{~dB}$, and $1.3 \mathrm{~dB}$, respectively. The smaller gains in symbol error rate when compared to bit error rate can be attributed to the bit-level correlation addressed by the correlation decoding component. In the conditional probability tables of the NB-JCCND, symbols where the majority of bits are not equal are discouraged by the correlation decoder component when correlation is $p_{1,2}>0.5$. Thus, all symbols are not equally likely to cause errors during decoding, and the most likely symbols to cause errors are those where the majority of bits are correct.

For some additional perspective on the gains achieved by NBJCCND, a system is considered which employs source coding across sensors. As a result of this source coding, each sensor will be able to reduce their respective data rates, and all bits transmitted by the sensors will be i.i.d. with $p_{1,2}=0.5$. In the network given in Figure 2, there are two sensors $S_{1}$ and $S_{2}$, each transmitting 1 bit per channel use. Thus, there are four possible transmissions given by $S_{1} S_{2}=00,01,10,11$. When the two sensors are uncorrelated (i.e., $p_{1,2}=0.5$ ), the entropy of the system is 2 bits per channel use. However, when $p_{1,2} \neq 0.5$, the entropy is

$$
H\left(S_{1}, S_{2}\right)=-2\left(\left(\frac{p_{1,2}}{2}\right) \log _{2}\left(\frac{p_{1,2}}{2}\right)+\left(\frac{1-p_{1,2}}{2}\right) \log _{2}\left(\frac{1-p_{1,2}}{2}\right)\right) .
$$

When $p_{1,2}=1.0$, the entropy is 1 bit per channel use, and by applying source coding across the sensors this allows an increase in the energy per bit by a factor of two. This translates to a gain in SNR given by

$$
\mathrm{SNR}_{\text {gain }}=10 \log _{10}(2.0)=3.0103 \mathrm{~dB}
$$

Thus, if source coding were applied across the sensors, and the NB-JNCD were used at the decoder, the gain would be $3.0103 d B$ when $p_{1,2}=1.0$. The source coding gains that could be achieved using $p_{1,2}=0.6,0.7,0.8$ and 0.9 is $0.0635 \mathrm{~dB}$, $0.2657 \mathrm{~dB}, 0.6501 \mathrm{~dB}$, and $1.3401 \mathrm{~dB}$, respectively. Each of these gains is comparable to those achieved using NB-JCCND, without the need for source coding across sensors.

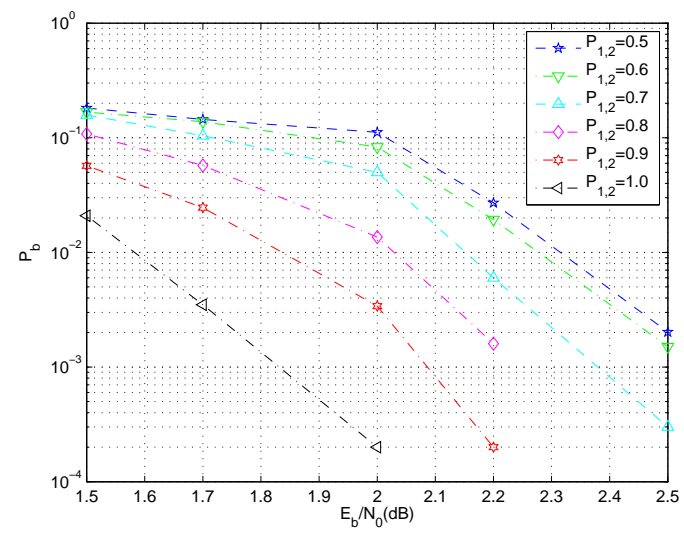

Fig. 6. Probability of bit error for NB-JCCND over the AWGN channel on a two-source, single-sink network under different bit-level correlations.

Simulation results are given in Figures 6 and 7 showing the performance of non-binary joint channel-correlation-network decoding over the non-fading additive white Gaussian noise 


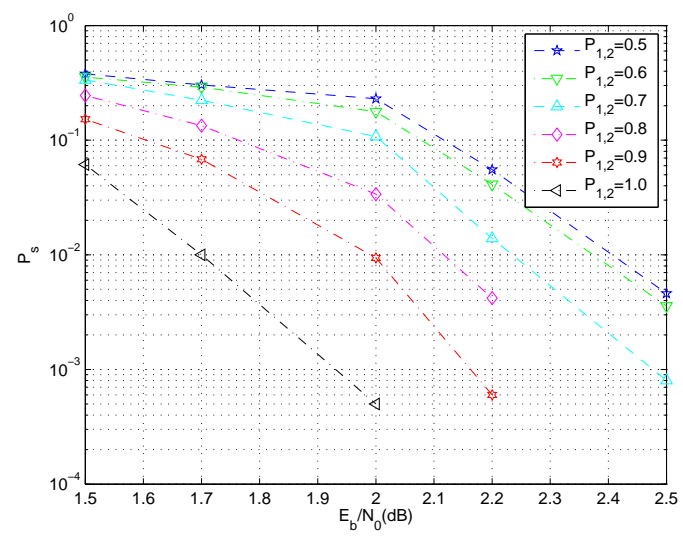

Fig. 7. Probability of symbol error for NB-JCCND over the AWGN channel on a two-source, single-sink network under different bit-level correlations.

channel. The gain of NB-JCCND over NB-JNCD $\left(p_{1,2}=0.5\right)$ at $P_{b}=10^{-2}$ for $p_{1,2}=0.6,0.7,0.8,0.9$, and 1.0 is $0.06 \mathrm{~dB}$, $0.18 \mathrm{~dB}, 0.31 \mathrm{~dB}, 0.42 \mathrm{~dB}$, and $0.69 \mathrm{~dB}$, respectively. The gains achieved with NB-JCCND over the AWGN channel are not as significant as those achieved over the Rayleigh block-fading channel. However, when compared to applying source coding across sensors, the gains are similar for low levels of correlation $p_{1,2} \leq 0.7$ without requiring costly sensor-to-sensor communications and additional processing at the sensor nodes.

\section{CONCLUSION}

This paper proposes the non-binary joint channel-correlationnetwork decoder, and demonstrates its performance over wireless sensor networks in which the sensors transmit correlated data. It is shown that when the correlation model is known at the decoder, a correlation-based decoding component inserted into the iterative process of the NB-JNCD significantly reduces the error rates. The new NB-JCCND has similar performance to a system that employs source coding across sensors to reduce the data rate and increase the available energy per bit. However, by exploiting the known correlation between sensors in the proposed correlation decoder component, NB-JCCND eliminates the need for costly sensor-to-sensor communications.

This paper examines decoding performance on a two-source, single-sink network. Future work includes testing NB-JCCND on a variety of networks with differing topologies. In networks that require decoding at the intermediate nodes, it is possible to incorporate correlation decoding with channel decoding, even under scenarios where network decoding is not possible. It is also of interest to examine different models of correlation such as a hidden Markov model, as its flexibility makes it suitable for a variety of applications, such as video surveillance [24].

While it was assumed in this paper that parity symbols are uncorrelated between sensor codewords, this is not generally true when the information symbols are correlated. In particular, low-density generator matrix codes are known to result in codewords with parity symbol correlation similar to that of the information symbol correlation [25]. By using the known correlation between parity symbols, the performance of NBJCCND would likely improve, especially when using moderateto-low rate LDPC codes.

\section{REFERENCES}

[1] R. Ahlswede, N. Cai, S. Li, and R. Yeung, "Network information flow," IEEE Trans. On Information Theory, vol. 46, pp. 1204-1216, July 2000.

[2] S. Katti, D. Katabi, W. Hu, H. Rahul, and M. Médard, "The importance of being opportunistic: Practical network coding for wireless environments.," in Proceedings of 43rd Allerton Conf. on Comm., Control, and Computing, no. September, (Monticello, IL), September 2005.

[3] S. Katti, H. Rahul, W. Hu, D. Katabi, M. Medard, and J. Crowcroft, "Xors in the air: Practical wireless network coding," Networking, IEEE/ACM Trans. on, vol. 16, pp. 497-510, June 2008.

[4] Y. Wu, P. A. Chou, and S. Kung, "Information exchange in wireless networks with network coding and physical-layer broadcast," Tech. Rep. MSR-TR-2004-78, Microsoft Research, Redmond, WA, August 2004.

[5] M. Ghaderi, D. Towsley, and J. Kurose, "Reliability gain of network coding in lossy wireless networks," in INFOCOM 2008. The 27th Conf. on Computer Comms.. IEEE, pp. 2171-2179, April 2008.

[6] A. Eryilmaz, A. Ozdaglar, and M. Medard, "On delay performance gains from network coding," in CISS, (Princeton,NJ), pp. 864-870, March 2006.

[7] D. Nguyen, T. Nguyen, and B. Bose, "Wireless broadcasting using network coding," in NetCod, (San Diego, CA), January 2007.

[8] I. Akyildiz, W. Su, Y. Sankarasubramaniam, and E. Cayirci, "A survey on sensor networks," Comms. Magazine, IEEE, vol. 40, pp. 102-114, August 2002.

[9] S. Marinkovic and E. Popovici, "Network coding for efficient error recovery in wireless sensor networks for medical applications," in 2009 Int'l Conf. on Emerging Network Intelligence, IEEE Computer Society, (Sliema, Malta), October 2009.

[10] M. Effros, T. Medard, M. Ho, S. Ray, D. Karger, and R. Koetter, "Linear network codes: A unified framework for source channel, and network coding," in 2003 DIMACS Workshop On Network Information Theory, (Piscataway, NJ), March 2003.

[11] J. Kliewer, T. Dikaliotis, and T. Ho, "On the performance of joint and separate channel and network coding in wireless fading networks," in Information Theory for Wireless Networks, 2007 IEEE Information Theory Workshop on, pp. 1-5, July 2007.

[12] C. Hausl and P. Dupraz, "Joint network-channel coding for the multipleaccess relay channel," in Int'l Workshop on Wireless Ad Hoc and Sensor Networks, vol. 3, pp. 817-822, September 2006.

[13] C. Hausl, F. Schreckenbach, I. Oikonomidis, and G. Bauch, "Iterative network and channel decoding on a tanner," in Proceedings of 43rd Allerton Conf. on Comm., Control, and Computing, (Monticello, IL), September 2005.

[14] X. Bao and J. Li, "Adaptive network coded cooperation (ancc) for wireless relay networks: matching code-on-graph with network-on-graph," Wireless Comms., IEEE Trans. on, vol. 7, pp. 574-583, February 2008.

[15] X. Bao and J. Li, "A unified channel-network coding treatment for user cooperation in wireless ad-hoc networks," in Information Theory, 2006 IEEE Int'l Symposium on, pp. 202-206, July 2006.

[16] Z. Guo, J. Huang, B. Wang, J. Cui, S. Zhou, and W. P., "Non-binary joint network and channel coding for underwater sensor networks," in 2008 The Int'l Workshop on UnderWater Networks, September 2008.

[17] Z. Guo, J. Huang, B. Wang, J. Cui, S. Zhou, and P. Willett, "A practical joint network-channel coding scheme for reliable communication in wireless networks," in Proceedings of the Int'l Symposium on Mobile Ad Hoc Networking and Computing, May 2009.

[18] H. Alemdar and C. Ersoy, "Wireless sensor networks for healthcare: A survey," Computer Networks, vol. 54, pp. 2688-2710, October 2010.

[19] R. G. Gallager, Low-Density Parity-Check Codes. PhD thesis, MIT, Cambridge, MA, 1960.

[20] D. J. C. MacKay and R. M. Neal, "Near shannon limit performance of low density parity check codes," Electronics Letters, vol. 33, pp. 457-458, March 1997.

[21] M. Davey and D. Mackay, "Low density parity check codes over gf(q)," IEEE Comms. Letters, vol. 2, pp. 165-167, June 1998.

[22] C. Fragouli, J. Le Boudec, and J. Widmer, "Network coding: an instant primer," SIGCOMM Comp. Comm. Rev., vol. 36, pp. 63-68, January 2006.

[23] J. Garcia-Frias, "Compression of correlated binary sources using turbo codes," Comms. Letters, IEEE, vol. 5, pp. 417-419, October 2001.

[24] J. Del Ser, M. Mendicute, P. Crespo, S. Gil-Lopez, and I. Olabarrieta, "Joint source-channel-network decoding and blind estimation of correlated sensors using concatenated zigzag codes," in Ad-Hoc, Mobile and Wireless Networks, pp. 30-41, Springer Berlin Heidelberg, 2009.

[25] A. Murugan, P. Gopala, and H. Gamal, "Correlated sources over wireless channels: cooperative source-channel coding," Selected Areas in Comms., IEEE Journal on, vol. 22, pp. 988 - 998, August 2004. 